\title{
Improving Health in an Integrated Health Care System: Empowering Pharmacists to Function as Key Agents of Change
}

\author{
Jennifer Langhinrichsen-Rohling1*, Donald Ponquinette ${ }^{2}$ \\ ${ }^{1}$ Director, Gulf Coast Behavioral Health and Resiliency Center, University of South Alabama \\ ${ }^{2} B S$ Pharmacy, $R P h$
}

Received: August 17, 2016; Accepted: September 14, 2016; Published: October 19, 2016

*Corresponding author: Jennifer Langhinrichsen-Rohling Ph.D, Director, Gulf Coast Behavioral Health and Resiliency Center, University of South Alabama, 600 Clinic Drive, TRP IV, Room 306,Mobile, AL 36688. Email: jlr@southalabama.edu.

\begin{abstract}
With passage of the Affordable Care Act (ACA) in 2010, the integrated health care model has become standard in the United States. Implementing integrated healthcare teams has been empirically shown to improve patient health outcomes and reduce health care costs system-wide. Pharmacists are positioned to play a pivotal role, especially as health educators, in this new model. However, several systemic and policy-related barriers may impede pharmacists from realizing their full potential in the new integrated healthcare world. This paper describes the evolving integrated health care system and pharmacists' potential role in it. Through the use of real-world case examples, we elucidate how promoting pharmacists' full participation is likely to positively affect patient health outcomes. We conclude with some policy considerations related to pharmacists' abilities to function as key agents of change within the integrated health care system.
\end{abstract}

\section{Introduction}

When the Affordable Care Act (ACA) became law in 2010, it affirmed the importance and efficacy of using an integrated health care model. It has also showcased new opportunities for pharmacists [11]. Practicing within an integrated care framework has been demonstrated to improve health outcomes and reduce health care costs [2]. The ACA promotes integrated care in several ways, through covering mental health and substance abuse treatment as "essential benefits"; offering new payment models, promoting patient-centered medical homes; increasing access to care; enhancing infrastructure; and providing new mechanisms and funding opportunities designed to facilitate coordinated and person-centered care [4].

The embracing of an integrated care approach, cemented by the ACA, is restructuring how we think about and practice healthcare in the United States. An integrated system unites and coordinates all aspects of health care, including primary care, mental health, and substance abuse. Although various versions of integrated health models have been articulated [9], each takes a holistic stance, recognizing that all types of health are interrelated and that health is affected by social determinants, including environment, education, and income. Integrated care privileges the voice of the patient and promotes coordination among providers to construct a plan that addresses the patient's multiple health needs. Fully enacted integrated health also includes a deliberate focus on preventive healthcare and health education [8].

The Gulf Coast region (Alabama, Mississippi, Florida, Texas, and Louisiana) has some of the worst health outcomes in the nation, high rates of chronic disease, high numbers of medically underserved and rural patients, and a shortage of practitioners [7]. It is also an area subjected to a large number of natural disasters [6]. With integrated care in mind, the National Institute for Minority Health and Health Disparities (NIMHD) funded seven Transdisciplinary Collaborative Centers to explore best practices for reducing health disparities. One of these, the Gulf States Health Policy Center, supports health policy research to improve health outcomes and reduce health disparities in the Gulf States. In its policy research work, the Gulf States HPC started ?developing a study to assess the impact of policies enacted to empower midlevel practitioners in the new integrated healthcare system, in ways designed to improve health outcomes and reduce health disparities. Efforts to empower mid-level practitioners has resulted in legislation that has been introduced nationally such as H.R. 592/S. 314, the Pharmacy and Medically Underserved Areas Enhancement Act [13]. Thus, the current paper describes a growing national focus on pharmacists as essential agents of change, particularly in providing integrated healthcare to underresourced individuals.

\section{Empowering Pharmacists}

All integrated healthcare models require that practitioners from multiple disciplines work together in a coordinated fashion. For integrated healthcare to work, all practitioners must know their role, respect the role of other practitioners, and participate in crafting and enacting a unified health care plan. The integrated 
model also works best when practitioners are empowered to practice at the highest level of their competency and capacity. Thus, empowering mid-level practitioners (nurse practitioners, physicians' assistants, and pharmacists) may be critical, particularly vis-a-vis preventive health education, much of which could be targeted toward effective treatment of expensive chronic diseases. Pharmacists may also be uniquely positioned to identify and address inappropriate medication use, by which the healthcare system incurred more than 177 billion dollars in costs in 2010 [5].

Pharmacists are positioned to play another pivotal role in the integrated care model; they may be ideal health educators. Pharmacists are trained in preventive health and cultural competency. They also have training in medication therapy management (MTM), which was expanded in the ACA [5]. In many cases, they may be more likely than doctors to have additional time to spend with patients, educating them not only about medication, but about nutrition, physical activity, and tobacco cessation. Pharmacists are also accessible: they are proximally located to patients, provide care after hours, and often work in their patients' home communities. Further, patients are more likely to have a familiar relationship with their local pharmacists, enhancing their comfort level. Pharmacists have consistently been ranked as one of the most trusted professionals, ranking second in 2014 [12]. Pharmacists can know their patients by their first name; they do not require an appointment and they tend to play a role in community health initiatives and events. According to a recent meta-analysis, including pharmacists on the health care team can be demonstrated to have direct patient impact via reduced hospital readmission and shorter days, as well as reductions in visits to the emergency room [3].

\section{The Barriers}

Unfortunately, several barriers are likely to prevent pharmacists from realizing their full potential in the new integrated health care model. Firstly, pharmacists do not have provider status nationwide [13], which often excludes them from a central role on the integrated care team. Unfortunately, pharmacists were excluded from the Social Security Act, which prevents many healthcare plans from compensating pharmacists for services including medication coordination [10]. Perhaps as a result, in many of the Gulf States, pharmacists do not have provider status and/or their scope of practice is legislatively limited. Payment for services, such as MTM, may also be limited or non-existent [13]. All of these systemic barriers discourage pharmacists from time spent on preventive health. Thirdly, pharmacists are often left out of electronic health information exchanges that allow practitioners to collaborate on patient care. Lastly, political barriers can prevent pharmacists from collaborative decision-making; doctors and other health professionals may sometimes be concerned about losing prestige, power, or funding if they accept pharmacists as equal partners on the care team.

These barriers can serve to negatively impact patient care, can hinder the sustainable success of integrated health care system, and dampen health outcomes. To illustrate these points, this paper describes three prototypical Gulf Coast patients who were impacted by the inclusion/exclusion of pharmacy in their overall care plan. These case studies draw upon patients cared for by the second author of this paper who has been a practicing neighborhood pharmacist in a high poverty, primarily African American area of Mobile, Alabama for the past 42 years. The following case studies represent amalgams of patients with whom this neighborhood pharmacist has worked.

\section{Case 1 -Overly Compliant Patient}

An African American woman in her early seventies, "Tonya" was diagnosed with gout by her primary care physician. After the diagnosis, she was prescribed common medications to relieve her discomfort and help manage her symptoms. As was her nature, Tonya was compliant to the doctor's order and took her prescription as directed. However, soon after starting the drugs, Tonya developed a terrible rash and experienced severe itching. Unfortunately, she continued to take her medications without question or consultation because her doctor had instructed her to do so. Out of respect, she did not want to question her doctor's judgment. Unfortunately, Tonya's symptoms continued to worsen, and within a few days, massive amounts of her skin began peeling off. By the time Tonya's family called her prescribing doctor for her, after consultation with and urging from the neighborhood pharmacist, it was too late. Tonya passed away. As it turned out, she was suffering from a rare side effect from her prescribed medication. Early and system-supported multi-disciplinary team communication, initiated by the pharmacist, who lived and worked in Tonya's neighborhood, might have facilitated a quicker return to her primary care provider, making an earlier change of treatment possible. This outcome might have also been averted through the active use of an electronic health record exchange that included pharmacy.

This case study highlights problems that can manifest in patients who are "overly compliant" with their primary care provider, perhaps because of the prestige physicians engender and/or the cost and time associated with obtaining additional medical care. Had this patient conversed with her neighborhood pharmacist, a practitioner who was readily accessible to her, when the side effects initially emerged, the pharmacist could have encouraged her to return to the physician for a re-diagnosis and/or facilitated an adjustment of her medication, before her side effects became life-threatening.

\section{Case 2 - Extensive Doctor Utilization}

An African American man in his early fifties, "George" experienced dizziness and a tingling sensation in his limbs. His physician recommended that he take blood pressure pills to regulate his condition and reduce his symptoms. The patient began the prescribed regimen. However, not fully trusting his original doctor, George chose to consult additional physicians for second and third opinions. Each doctor was located in a different clinic and none were connected via a health information exchange. Eventually, George was being treated for the same symptoms by four separate physicians. Unfortunately, he ended 
up being prescribed four different drugs as a result. Luckily, this situation was noted by his neighborhood pharmacist. After an on-site medication consult, George's care was stream-lined and improved. A costly medication error was averted.

This case highlights the problems that can occur with patients who are noncompliant with their primary physician-administered care plan, drug seeking, or who get multiple doctors involved in their care without collaborative communication. Dangerous drug interactions and potential overdoses are risks that an empowered pharmacist can catch and address.

Patients may also be non-compliant with the medication, in ways that promote adverse health outcomes. There are numerous reasons why under-resourced patients may fail to take their medications properly. Patients can be confused about the required dosage to achieve impact. In high poverty areas, it is not uncommon for patients to stretch their medication to make it last longer. When money is tight, patients sometimes choose to not fill their prescriptions. Some undereducated patients have been found to take a generic and a brand name of the same drug without realizing it. These issues are often rooted in socioeconomic factors, including low literacy levels, financial stress, food insecurity, and lack of transportation. Pharmacists are uniquely poised to address these issues with patients, and they often have a deep understanding of contributing socioeconomic factors that are prevalent in the surrounding neighborhoods and communities. However, without being reimbursed for consultation activities, pharmacists are limited in the time they can spend addressing issues that can have serious impacts on health and indeed, are often the root cause of poor health outcomes.

\section{Case 3 - Displaced by Disaster}

Following the aftermath of Hurricane Katrina, thousands were displaced from their homes. In Alabama, displaced New Orleans residents arrived with no clothes, no money, and, too often insufficient medications and health information. When trying to refill prescriptions, refugee patients often could only describe the shape and color of the pills they had been taking. The names of the medications were unknown to the patients, and, for some, there was a lack of knowledge of the conditions for which they were being treated.

Specifically, immediately after Katrina, "Leroy" was in need of medication. Leroy was a 61 year old white male with low literacy and poor education. He indicated to the neighborhood pharmacist that he took this medication daily and if he didn't take it, he would end up in the hospital. While Leroy could describe the shape and color of the pill he took, he didn't know the name of the medication nor the illness for which it was prescribed. No electronic information related to Leroy's health was available.

Fortunately for Leroy, his new neighborhood pharmacist spent time with Leroy and, after consultation and permission; their conversation was broadened to include members of Leroy's immediate family. It was determined that Leroy suffered from paranoid schizophrenia. He had experienced numerous previous hospitalizations and had a history of homelessness. With effort from the pharmacy group, Leroy was helped to get access to a primary care physician who quickly re-prescribed the appropriate medication. Leroy successfully avoided rehospitalization post-disaster.

This case highlights problems that can be experienced in areas of the country with poor healthcare systems, lack of electronic health information exchanges - both within and across states, and a propensity for natural disasters. Integrated health professionals must know the medications that patients are taking, even when they have relocated from another part of the state or another region in the country. This shared knowledge may only be possible if medication history is located in a widely accessible electronic system. However, in many underserved areas, pharmacists have not yet been included in this initiative, which leaves patients, already vulnerable because of disaster, potentially more at-risk.

\section{Policy Considerations}

1. Taking these cases into account, some changes could be considered that would empower pharmacists to function more effectively within the new integrated health care model:Pharmacists could be assured of provider status, which would make pharmacy consultation a billable and reimbursable expense. This would help pharmacists have a stronger voice within integrated care and a deliberate place in electronic health information exchanges.

2. Pharmacists could be incentivized and rewarded for practicing in medically underserved areas through student loan forgiveness programs comparable to those physicians and dentists receive.

3. A federal Collaborative Drug Therapy Act could be advanced in order to make pharmacists' ability to collaborate with the integrated care team consistent across states.

4. Cultural competency could be a privileged part of accredited pharmacy programs to ensure that the trusted relationship between pharmacists and their diverse patients continues and flourishes.

These possibilities could help ensure that the new integrated health care model, embraced by the ACA, will be maximally effective in improving health outcomes. Evidence indicates that a robust inclusion of pharmacists within the integrated health system willower health costs and reduces health disparities by empowering pharmacists to practice at their highest capability. However, the direct impact of any enacted policy changes requires careful evaluation, such as what can be undertaken by the Gulf States Health Policy Center. Located within their home communities, pharmacists are poised to take an active role in improving under-resourced patients' access to essential health education, while preventing adverse health events and enhancing community resiliency. 


\section{References}

1. America's Health Rankings. United Health Foundation. 2016.

2. Blumenthal D, Abrams M, Nuzum R. The Affordable Care Act at 5 Years.New England Journal of Medicine. 2015;372(25):2451-2458. doi: 10.1056/NEJMhpr1503614.

3. Chisholm-Burns MA, Kim Lee J, Spivey CA, Slack M, Herrier RN, HallLipsy E, et al. US pharmacist's effect as team members on patient care: Systematic review and meta-analysis. 2010;48(10):923-933. doi: 10.1097/MLR.0b013e3181e57962.

4. Croft B, Parish SL. Care integration in the Patient Protection and Affordable Care Act: Implications for behavioral health.Administration and Policy in Mental Health. 2013;40(4):258-263. doi: 10.1007/ s10488-012-0405-0.

5. Diagle L. Pharmacists' role in accountable care organizations. American Society of Health-System Pharmacists. 2011;1-7.

6. Gohres A. Why Does the Gulf of Mexico Need a Disaster Response Center?" National Oceanic and Atmospheric Administration. 2011
7. Shortage Designation: Health Professional Shortage Areas \& Medically Underserved Areas/Populations. HRSA. 2016.

8. Koh HK, Sebelius KG.. Promoting prevention through the Affordable Care Act. New England Journal of Medicine. 2010;363(14):1296-1299. doi: 10.1056/NEJMp1008560.

9. Langhinrichsen-Rohling J, Wornell C, Johns K, Selwyn C, \& Friend J The nuts and bolts of developing integrated healthcare in underresourced primary care settings: Challenges \& lessons learned. In W. S. Craig (Ed.), Integrated psychological services in primary care. Hauppauge, NY: Nova Science Publishers. 2015;67-87.

10. Marotta R. The state of provider status: An update for pharmacy students. Pharmacy Times. 2016.

11. Matzke GR. Health care reform : Opportunities for pharmacists. Annals of Pharmacotherapy. 2012;46(4):27-32. doi: 10.1345/aph.1Q803.

12. National Association of Boards of Pharmacy. Pharmacists ranked as second most trusted profession in annual Gallop poll. Pharmacy times. 2016

13. Yap D. Provider status efforts, successes presented at APhA2016. Pharmacy today. 2016 